\section{Infecção latente por tuberculose entre pessoas com HIVIAIDS, fatores associados e progressão para doença ativa em município no Sul do Brasil}

\section{Latent tuberculosis infection in persons with HIV/AIDS, associated factors, and progression to active disease in a city in southern Brazil}

\section{Infección latente por tuberculosis entre personas con VIH/SIDA, factores asociados y progresión para una enfermedad activa en un municipio del sur de Brasil}

Danielle Talita dos Santos 1

Maria Concebida Garcia 1

Arlete Alves Nunes Fragoso da Costa 2

Flávia Meneguetti Pieri 3

Denise Andrade Pereira Meier 3

Silvia Paulino Ribeiro Albanese 3

Ricardo Alexandre Arcêncio 1

Elma Mathias Dessunti 3

\section{Resumo}

Propôs-se levantar a prevalência de infecção latente por tuberculose (ILTB) entre pessoas vivendo com HIV/AIDS (PVHA), fatores associados e se entre os casos identificados houve progressão para tuberculose ativa. Trata-se de estudo epidemiológico e descritivo. A população foi composta de PVHA, atendidas entre 2003 e 2014 em um centro de referência para HIV/AIDS. Os dados foram coletados com base em prontuários e fichas do Sistema de Informação de Agravos de Notificação (SINAN). Procedeu-se à estatística bivariada, com aplicação do teste qui-quadrado, em que as variáveis com valores de $p<0,2$ foram selecionadas para entrar no modelo de regressão múltipla. Foi fixado em todos os testes o erro tipo I em 5\% ( $p<0,05)$. No estudo, 690 casos foram analisados, sendo que $66(9,4 \%)$ apresentaram o diagnóstico de ILTB, tendo uma prevalência de 7,5 casos para cada 100 pacientes. Dos 53 (80,3\%) casos de ILTB que tiveram o tratamento indicado com isoniazida, apenas 26 $(39,4 \%)$ concluíram e $10(15,1 \%)$ abandonaram. Observou-se que as variáveis sexo masculino (OR ajustado = 1,8; IC95\%: 1,1-3,3), situação prisional (OR ajustado = 7,6; IC95\%: 2,35-24,9) e contagem de linfócitos mais altos são fatores associados ao diagnóstico de ILTB (OR ajustado = 1, 1; IC95\%: 1,1-1,2). Verificou-se que $47(6,7 \%)$ dos casos de ILTB progrediram para TB ativa. $O$ diagnóstico e o tratamento de ILTB nas PVHA não foram priorizados, o que contribuiu para o desenvolvimento de doença ativa entre os casos. $O$ trabalho contribuiu para o avanço do conhecimento acerca da ILTB entre PVHA, demonstrando aspectos cruciais no que tange ao manejo de PVHA e ainda a importância da detecção da ILTB e a instituição precoce da isoniazida, visando à melhor qualidade de vida e prognóstico das PVHA.

Teste Tuberculinico; Tuberculose Latente; Tuberculose;

Sindrome de Imunodeficiência Adquirida; HIV

\section{Correspondência}

D. T. Santos

Av. Caramuru 2600, bloco 12, apto. 12, Ribeirão Preto, SP 14040-902, Brasil.

danielletalita@hotmail.com

1 Escola de Enfermagem de Ribeirao Preto, Universidade de São Paulo, Ribeirão Preto, Brasil.

2 Secretaria Municipal de Saúde, Londrina, Brasil.

3 Universidade Estadual de Londrina, Londrina, Brasil. 


\section{Introdução}

A tuberculose (TB) é uma das principais causas de óbito entre pessoas vivendo com HIV/AIDS (PVHA). Esses casos têm de 26 a 31 vezes mais riscos de adoecimento e óbito por TB do que pessoas não infectadas 1. Estima-se que, no mundo, morreram 1,5 milhão de pessoas por TB, sendo que 400 mil eram PVHA 2,3. No Brasil, houve crescimento da incidência da coinfecção TB/HIV, demonstrado pelos dados de confirmação do diagnóstico de HIV em 9,8\% das pessoas com TB que foram testadas 4 .

Aproximadamente um terço da população mundial está infectada pelo Mycobacterium tuberculosis 2,3, ou seja, com a infecção latente da tuberculose (ILTB). Isso, todavia, não significa que todos adoecerão com a forma ativa, senão que constituem reservatórios do bacilo que podem ser reativados sob condições de resposta imunológica deficiente, como é o caso das PVHA 2,3.

Diante da relevância da ILTB na manutenção da transmissão da TB, é importante o seu diagnóstico em PVHA, o que no Brasil é feito prioritariamente pela interpretação da prova tuberculínica (PT) em função do cenário epidemiológico em pacientes sem evidências de TB ativa 5. O diagnóstico da ILTB permite indicar a implantação do tratamento com isoniazida e, consequentemente, reduzir o risco de desenvolvimento da doença ativa, cujo tratamento é mais complexo, oneroso e com chances de prognóstico desfavorável 6,7,8.

Para o diagnóstico da ILTB entre as PVHA, destaca-se a utilização da PT que é recomendada desde 1998 pela Organização Mundial da Saúde (OMS) e pelo Programa Conjunto das Nações Unidas sobre HIV/AIDS (UNAIDS) e adotada pelo Brasil no ano seguinte, porém, sua aplicação é baixa 7,8. Assim, a PT ainda é o exame recomendado para o diagnóstico da ILTB, porém, pesquisas vêm comparando o exame interferon gamma release assay (IGRA) para a sua detecção e apontam que ambos os testes apresentam sensibilidade subótima, especialmente em indivíduos severamente imunocomprometidos 1,9 .

Visando a combater a coinfecção TB/HIV, a OMS propôs os três Is: Intensified Case Finding (ICF), Isoniazid Preventive Therapy (IPT) e TB Infection Control (IC) 8, com ênfase na terapia preventiva com isoniazida como uma ação central para os serviços especializados na atenção às PVHA 10.

No que tange aos fatores associados à ILTB em PVHA, alguns estudos apontam o etilismo 11, o uso de drogas injetáveis 12 , estágio avançado do HIV, ambientes insalubres e gênero feminino 13. Outra questão emblemática diz respeito à instituição do tratamento entre os casos de ILTB; na literatura há relatos de pacientes que, mesmo com diagnóstico de ILTB, desenvolveram TB ativa, pois não foram indicados à terapia com isoniazida 14; outro fator determinante para adoecimento das PVHA é a não instituição precoce e uso regular da terapia antirretroviral (TARV), sendo que esta terapia pode reduzir em até $51 \%$ as chances de desenvolver a TB ativa 15 .

Embora esse seja um objeto de grande importância para as políticas públicas e para o avanço do conhecimento acerca da cadeia de progressão da TB e para a qualidade de vida das PVHA no Brasil, observam-se poucos estudos com este enfoque. Outra lacuna é a inexistência de trabalhos que avaliem o horizonte dos pacientes diagnosticados com ILTB, seja em termos clínicos, epidemiológicos ou operacionais. Assim, o presente estudo tem como objetivo levantar a prevalência de ILTB entre PVHA, fatores associados e se entre os casos identificados houve progressão para TB ativa.

\section{Método}

\section{Tipo de estudo}

Trata-se de um estudo epidemiológico descritivo 16.

\section{Cenário de estudo}

O trabalho foi realizado em um centro de referência, que está situado no Município de Londrina, Estado do Paraná, Brasil, que apresentou incidência de TB de 20,5 casos por 100 mil habitantes e incidência de HIV de 17,7 casos por 100 mil habitantes 4,17 no ano de 2014.

No referido ano, 91\% dos casos detectados com TB foram testados para HIV. Entre os casos com baciloscopia positiva, apenas $69 \%$ concluíram o tratamento (abaixo da meta estabelecida pela OMS, 
que é de 85\%) 4. Quanto aos casos com HIV, 27,1\% dos detectados apresentaram contagem de linfócitos TCD4+ inferior a 200 células $/ \mathrm{mm}^{3}$ na ocasião do diagnóstico, o que denota diagnóstico tardio. Em termos de mortalidade por HIV, em 2015, a Regional de Saúde de Londrina apresentou 4,8 óbitos por 100 mil habitantes 17 .

Sobre o centro de referência estudado, este conta com dois serviços de atendimento especializado: o setor de pneumologia que é voltado à atenção dos pacientes com TB e respectivos contatos; e o ambulatório que atende às PVHA, este último conta com um total de 4.010 pessoas com HIV cadastradas desde o início de suas atividades, sendo responsável pelo atendimento de 21 municípios da região norte do Paraná.

\section{População de estudo}

Essa população foi composta por PVHA atendidas no período de 2003 a 2014. Foram excluídos os casos em que os prontuários não foram encontrados, os que não retornaram para a leitura da prova tuberculínica e aqueles com diagnóstico de TB ativa anterior à primeira PT (Figura 1).

\section{Variáveis de estudo e coleta de dados}

Para o levantamento dos dados foi criado um instrumento contendo informações sociodemográficas, registros sobre a PT (resultado e datas de repetição do exame), contagem de linfócitos TCD4+, data do diagnóstico do HIV, uso da terapia antirretroviral, data de início do acompanhamento no ambulatório de HIV e dados sobre o diagnóstico e tratamento da ILTB ou TB ativa. Os elementos considerados pela equipe do centro de referência para o diagnóstico de TB ativa foram pautados em critérios laboratoriais, clínicos, radiológicos e epidemiológicos, segundo as diretrizes do Programa Nacional de Controle da Tuberculose (PNCT) do Ministério da Saúde 18 e a informação registrada no prontuário e no Sistema de Informação de Agravos de Notificação (SINAN).

As PVHA submetidas à PT foram elencadas baseando-se nos livros de registro do setor de pneumologia e, num segundo momento, procedeu-se à busca dos prontuários no ambulatório de HIV/ AIDS do centro de referência. Para a complementação dos dados recorreu-se também ao SINAN e aos prontuários do setor de pneumologia dos casos que apresentaram ILTB e TB.

Quando identificados os casos de ILTB ou de progressão da ILTB para a TB ativa, os pesquisadores buscaram também informações sobre os desfechos: se em tratamento, alta por cura, óbito ou abandono. A coleta de dados ocorreu entre os anos de 2012 e 2014 e contou com a participação de quatro recursos humanos com formação na área da saúde, que receberam treinamento prévio.

\section{Análise dos dados}

Essa análise foi realizada no programa SPSS, versão 20.0 (IBM Corp., Armonk, Estados Unidos). Foi aplicado o teste qui-quadrado com dicotomização das variáveis categóricas e cálculo da medida de associação do odds ratio (OR) e respectivo intervalo de 95\% de confiança (IC95\%). As variáveis foram submetidas à regressão logística simples, com obtenção do IC95\%. Na variável contínua contagem de células TCD4+, nos casos em que os pacientes tivessem mais de um exame, foi considerado o exame coletado mais recentemente e próximo à PT.

Ainda para essa variável, foi aplicado o teste não paramétrico Mann-Whitney para a obtenção de média, mediana e desvio padrão, e só então os dados foram submetidos à regressão logística simples para a obtenção de valor de OR bruto e IC.

As variáveis com valores de $\mathrm{p}<0,2$ foram selecionadas para entrar no modelo de regressão múltipla com a estratégia de stepwise manual backward-forward. Utilizou-se o valor da função de verossimilhança e o teste de Hosmer-Lemeshow para avaliar a significância e a qualidade do modelo. Em todos os testes foi fixado o erro tipo I em $5 \%(\mathrm{p}<0,05)$. 


\section{Aspectos éticos}

Esta pesquisa foi aprovada pelo Comitê de Ética em Pesquisa (CAAE no 4674.0.000.268-0) da Universidade Estadual de Londrina.

\section{Resultados}

Foram identificados 880 casos de pacientes com HIV atendidos no serviço onde realizaram a PT. Desses, 190 (21,6\%) foram excluídos por não apresentarem resultado da PT conhecida $(\mathrm{n}=69)$, por não terem o seu respectivo prontuário localizado $(n=113)$ e por apresentarem diagnóstico de TB no início do atendimento $(\mathrm{n}=8)$ (Figura 1). Foram confirmados $66(9,6 \%)$ casos de ILTB (Figura 1), o que representa uma prevalência de 7,5 casos para cada 100 pacientes.

Entre os casos de ILTB, 53 (80,3\%) tiveram o tratamento indicado com isoniazida, todavia, 14 $(26,4 \%)$ não iniciaram o tratamento. Dentre os 39 casos que iniciaram o tratamento, $26(66,6 \%)$ concluíram, 10 (25,6\%) abandonaram e outros $3(7,6 \%)$ casos não tiveram o desfecho conhecido pela falta de registros. Outro dado importante é que entre os casos de ILTB que abandonaram o tratamento, a média foi de 2,7 meses (mínimo de um e máximo de cinco meses) até o abandono.

Observou-se que 78 (11,3\%) PVHA nunca realizaram a PT com uma contagem maior que 200 células $/ \mathrm{mm}^{3}$, ocorrência atribuída à própria condição clínica do paciente que não apresentou restabelecimento na contagem de células durante o acompanhamento pelo serviço de saúde.

Verificou-se que ser do sexo masculino (OR ajustado = 1,8; IC95\%: 1,1-3,3), estar em situação prisional (OR ajustado = 7,6; IC95\%: 2,35-24,9) e a contagem de linfócitos elevada foram fatores associados à ILTB (OR ajustado = 1,1; IC95\%: 1,1-1,2) em PVHA (Tabela 1).

Evoluíram para a forma ativa 47 (6,8\%) PVHA, sendo que 13 (27\%) pessoas apresentaram diagnóstico de ILTB anterior, porém não foram encontrados registros de tratamento com isoniazida. Sobre os casos que evoluíram para TB ativa, 26 (55,3\%) registraram a forma clínica pulmonar (bacilífera), 6 $(12,8 \%)$ a forma miliar, $4(8,5 \%)$ ganglionar e $11(23,4 \%)$ apresentaram outras formas como intestinal, ocular e meningoencefálica.

Com relação ao tratamento da TB, 29 (61,7\%) pessoas realizaram o tratamento completo tendo recebido alta por cura, $13(27,6 \%)$ abandonaram o tratamento e em 5 (10,6\%) casos o tempo não pôde ser determinado por falta desta informação em prontuário e na ficha do SINAN (Figura 1). Houve 19 (2,7\%) casos de óbitos na população estudada, sendo que apenas 2 (0,3\%) estavam registrados no SINAN tendo a TB como causa básica.

\section{Discussão}

O estudo teve como objetivo levantar a prevalência de ILTB entre pacientes com HIV, os fatores associados e se, entre os casos identificados, houve progressão para TB ativa. Observou-se uma prevalência de 7,5 casos para cada 100 pacientes, registrando-se que ser do sexo masculino, estar em situação de privação de liberdade e contagem de linfócito TCD4+ mais elevada foram associados à ILTB; quanto à progressão para TB ativa, verificou-se que 47 (6,8\%) desenvolveram a doença.

Observou-se que 9,6\% da amostra foram diagnosticados com ILTB. Dados próximos foram mencionados em outros estudos no Brasil, de 9,4\% no Sudeste 19, 11,9\% no Sul 20 e mais alto no Nordeste 21 com 21,5\%. Na Índia, país que apresenta as mais elevadas taxas de incidência da TB no mundo, houve 64,3\% de ILTB diagnosticada entre PVHA 22; tais achados podem estar associados à alta prevalência da TB nessas localidades 23 .

Considerando-se a história natural da doença, a fase assintomática é prelúdio da fase sintomática. Dessa forma, o percentual de ILTB pode ser reflexo da presença da TB e os resultados encontrados no presente trabalho justificam-se devido ao cenário da pesquisa 19,22. O município do estudo foi considerado prioritário para o controle da TB pelo Ministério da Saúde quando apresentava incidência de 27,6 casos por 100 mil habitantes em 2006; em 2014 o mesmo foi removido da lista de prioritários por atender as metas de controle da doença e ter atingido uma incidência de 20,6 casos por 100 mil habitantes 4,24. 


\section{Figura 1}

Evolução para infecção latente da tuberculose (ILTB) e doença ativa entre os pacientes com HIV/AIDS acompanhados no centro de referência. Londrina, Paraná, Brasil, 2003-2014.

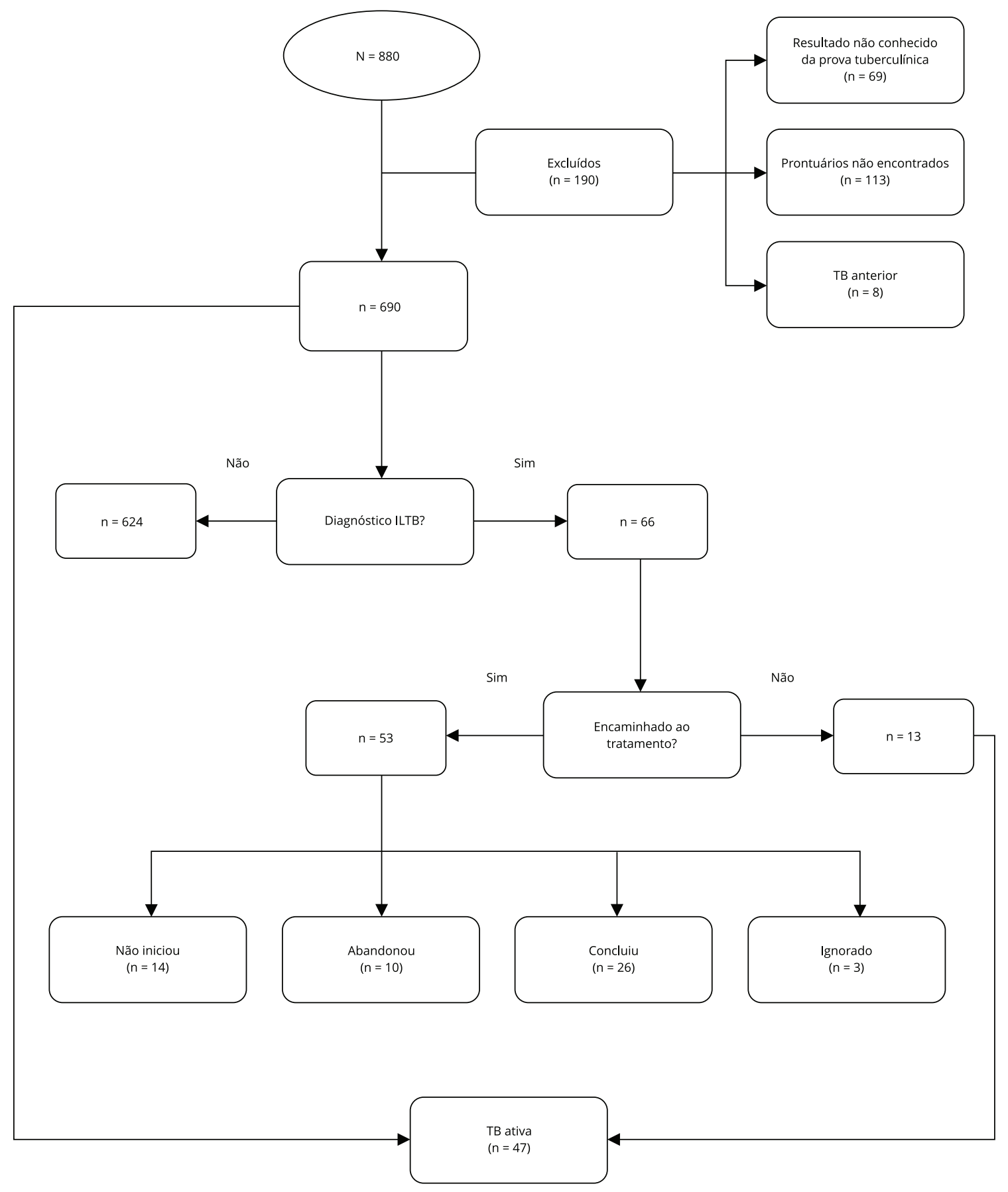


Fatores associados à infecção latente de tuberculose (ILTB) entre pessoas com HIV/AIDS atendidas em um centro de referência. Londrina, Paraná, Brasil, 2003-2014.

\begin{tabular}{|c|c|c|c|c|c|c|c|c|c|c|}
\hline \multirow[t]{3}{*}{ Variáveis sociodemográficas } & \multicolumn{4}{|c|}{ ILTB } & \multicolumn{3}{|c|}{ Análise bivariada } & \multicolumn{3}{|c|}{ Regressão múltipla } \\
\hline & \multicolumn{2}{|c|}{ Sim } & \multicolumn{2}{|c|}{ Não } & \multirow[t]{2}{*}{ OR bruto } & \multirow[t]{2}{*}{$\mathrm{IC95 \%}$} & \multirow[t]{2}{*}{ Valor de p } & \multirow[t]{2}{*}{ OR ajustado } & \multirow[t]{2}{*}{ IC95\% } & \multirow[t]{2}{*}{ Valor de $p$} \\
\hline & $\mathbf{n}$ & $\%$ & $\mathbf{n}$ & $\%$ & & & & & & \\
\hline \multicolumn{11}{|l|}{ Sexo $(n=690)$} \\
\hline Feminino & 17 & 6,1 & 260 & 93,9 & 1,0 & & & 1,0 & & \\
\hline Masculino & 49 & 11,9 & 364 & 88,1 & 2,1 & $1,1-3,6$ & 0,012 & 1,8 & $1,1-3,3$ & 0,048 \\
\hline \multicolumn{11}{|l|}{ Faixa etária em anos ( $n=687$ ) } \\
\hline $13-38$ & 28 & 8,0 & 324 & 92,0 & 1,0 & & & & & \\
\hline 39 e mais & 38 & 11,3 & 297 & 88,7 & 1,5 & $0,8-2,5$ & 0,132 & - & - & - \\
\hline \multicolumn{11}{|l|}{ Raça/Cor $(n=635)$} \\
\hline Branca/Amarela & 40 & 8,0 & 460 & 92,0 & 1,0 & & & & & \\
\hline Afrodescendente & 16 & 11,9 & 119 & 88,1 & 1,6 & $0,8-2,8$ & 0,161 & - & - & - \\
\hline \multicolumn{11}{|l|}{ Escolaridade em anos $(n=683)$} \\
\hline 8 e mais & 19 & 10,9 & 156 & 89,1 & 1,0 & & & & & \\
\hline $0-7$ & 47 & 9,3 & 461 & 90,7 & 1,2 & $0,7-2,1$ & 0,535 & - & - & - \\
\hline \multicolumn{11}{|l|}{ População prisional ( $n=678$ ) } \\
\hline Não & 57 & 8,6 & 609 & 91,4 & 1,0 & & & & & \\
\hline Sim & 5 & 41,7 & 7 & 58,3 & 7,6 & $2,3-24,8$ & $<0,0001$ & 7,3 & $2,2-24,5$ & 0,001 \\
\hline Contagem de células TCD4+ & \multirow{2}{*}{\multicolumn{2}{|c|}{$\begin{array}{c}527,79 \\
(294,37)\end{array}$}} & \multirow{2}{*}{\multicolumn{2}{|c|}{$\begin{array}{c}431,2 \\
(267,9)\end{array}$}} & 1,1 & $1,1-1,2$ & 0,006 & 1,1 & $1,1-1,2$ & 0,003 \\
\hline [média (DP)] & & & & & & & & & & \\
\hline
\end{tabular}

DP: desvio padrão; IC95\%: intervalo de 95\% de confiança; OR: odds ratio.

Nota: Hosmer-Lemeshow $=7,18$ e $p=0,517$.

Chama a atenção que o tratamento da ILTB tenha sido despendido a apenas 39 (59\%) dos indivíduos com esta infecção, bem como o abandono ter chegado a $25,6 \%$, visto que estudos apontam o início do tratamento em 69,8\% das pessoas elegíveis 11 e abandono de 2,2\% 5, resultados melhores quando comparados aos do presente trabalho. Ressalta-se que $13(19,7 \%)$ indivíduos com diagnóstico de ILTB evoluíram para TB ativa. Quando a terapia com isoniazida é adotada tem-se a redução substancial das chances de desenvolvimento da doença e do óbito 6,25,26.

O não início ou o abandono do tratamento da ILTB pode estar ligado a diversos fatores como o acolhimento e vínculo com a unidade onde o paciente é acompanhado, o grau de escolaridade e o uso de drogas 11, além do estágio avançado da infecção pelo HIV e ambientes insalubres 13 . A detecção e tratamento da ILTB, principalmente nos grupos populacionais com maiores chances de desenvolverem a forma ativa, funcionam como aliados estratégicos para o controle da transmissão 23,27, pois a condição de latência do bacilo torna o indivíduo um reservatório do mesmo, exercendo um importante papel na manutenção da cadeia de transmissão da TB 28.

Outra questão importante que pode ser levantada diante dos resultados do estudo refere-se à baixa adesão das equipes de saúde à investigação de ILTB entre PVHA e o não monitoramento longitudinal e sistemático dos casos em que o tratamento com isoniazida é prescrito. Assim, por não haver uma orientação ou protocolo de busca ativa aos pacientes no serviço, estas ações ficam por demais prejudicadas, resultados estes que podem estar corroborando para os indicadores epidemiológicos de taxas de mortalidade por HIV e TB.

Seria muito interessante a introdução de indicadores de monitoramento com percentual de casos de ILTB investigados entre PVHA, proporção de casos que concluíram o tratamento de ILTB, bem como o percentual de casos que desenvolveram TB ativa, haja vista que expressam a qualidade das ações desempenhadas pelos programas de saúde. Interferindo nesses indicadores, muito provavelmente se contribuiria à mudança do cenário epidemiológico encontrado 25,29. 
A necessidade de repetição da PT após o início da TARV justifica-se pela possibilidade da restauração imunológica do indivíduo com consequente melhora da resposta ao exame ${ }^{23,30}$. Pessoas com AIDS em estágio avançado (contagem de linfócitos TCD4+ $\leq 200$ células/ $/ \mathrm{mm}^{3}$ ) e com PT negativa deveriam ser submetidas à nova prova assim que iniciassem a TARV ou após o restabelecimento da contagem de linfócitos 30. Dessa forma, medidas substanciais precisariam ser efetuadas para que a PT pudesse ser utilizada com maior frequência entre PVHA, considerando-se ainda a superação de alguns desafios da atualidade como a redução do fornecimento do derivado protéico do M. tuberculosis (PPD) no país.

O exame imunológico IGRA vem sendo utilizado por diversos países para diagnosticar a ILTB, todavia, não há consenso sobre a superioridade do mesmo, além de seu elevado custo quando comparado à PT 9,23,29,31.

Diante das limitações da distribuição e até falta do PPD, existe um guideline 8 que traz recomendações para o tratamento da ILTB para PVHA; o tratamento, mesmo na ausência da PT, é fortemente recomendado devendo ser iniciado após o descarte do diagnóstico da forma ativa 7 .

Para que sejam cumpridas as recomendações preconizadas, a gestão do serviço local deve ser fortalecida com um sistema de registro com a máxima completude e uma sistemática vigilância e acompanhamento longitudinal dos casos. Entende-se como igualmente importantes para o sucesso de um programa ou ação em saúde o suprimento de materiais necessários, trabalhadores sensíveis e financiamento que garantam a execução dessas políticas 10,29,32,33.

Os resultados do estudo associam pessoas do sexo masculino, população prisional e número mais elevado de linfócitos TCD4+ à ILTB. De fato, observa-se que as duas infecções, TB e HIV/AIDS, apresentam predominância entre o gênero masculino 34,35 , semelhantemente à prevalência da ILTB que tem sido elevada neste gênero 11,12 . Os resultados podem ser justificados devido a, frequentemente, os homens serem associados à menor adoção de ações de promoção e prevenção em saúde e, por sua parte, menor procura por serviços de saúde 36 , vindo a adoecer e até morrer por doenças como a TB e AIDS 34,35.

Destaca-se o resultado de associação da ILTB junto à população prisional (IC > 1). É conhecida a vulnerabilidade de desenvolvimento da TB nas pessoas privadas de liberdade, explicado por razões tais como o ambiente superlotado e contato íntimo constante, que favorece a transmissão do bacilo 37,38. Estudos com essas pessoas apresentaram percentuais elevados de ILTB, $40 \% 39$ e 46,9\% 40 quando submetidas à PT. No país, autores apontam $61,5 \%$ no Nordeste $41,22,5 \%$ no Centro-oeste 42 e 73\% no Sudeste de prevalência de ILTB 43.

As políticas públicas de controle da TB para pessoas privadas de liberdade recomendam a investigação clínica, bacteriológica e radiológica na presença de sintomas como tosse por mais de duas semanas para diagnosticar a doença ativa, no entanto, não foi encontrada recomendação voltada à busca ativa e tratamento de ILTB neste público, apesar de estar em grande risco 18,24.

A variável contagem de linfócitos TCD4+ apresentou associação significativa com a ILTB (IC95\%: 1,1-1,2). Contagens mais elevadas dessas células proporcionam respostas mais efetivas da PT contrariamente à condição da anergia cutânea 44 , que ocorre quando a contagem de linfócitos TCD4+ está em níveis muito baixos 45 , favorecendo o resultado falso negativo 19,31 . Assim, mesmo que o paciente esteja infectado pode não responder adequadamente ao exame.

Uma possibilidade para a minimização dos efeitos da anergia cutânea em relação aos exames seria o monitoramento da contagem de linfócitos TCD4+ do paciente no momento ou próximo à realização da PT, assim como o controle das repetições do exame, quando necessário, para que se garantam resultados mais fidedignos 30,31,44. Observa-se que alguns pacientes nunca apresentaram melhora na contagem de linfócitos, o que ratifica a importância de novos métodos de diagnóstico da ILTB, muito embora seja esta uma área com poucos avanços na ciência 9 .

Dentre os 47 indivíduos que evoluíram para TB, verificamos que 13 (27,6\%) tiveram uma PT positiva e não foi possível confirmar se houve ou não tratamento de ILTB anteriormente ao desfecho da doença ativa. Outro resultado é que dentre os pacientes em tratamento para TB ativa, apenas 29 $(61,7 \%)$ concluíram, valores aquém das recomendações da OMS, que são de $85 \%$ 3,18.

Tais achados reforçam a importância de um trabalho sistemático e minucioso das equipes de saúde, haja vista que elas estão trabalhando com um grupo em risco elevado de morte que são as PVHA, portanto, os registros assim como todo o processo de trabalho devem ser ressignificados em sua essência. 
É importante ressaltar que os registros, fonte dos dados desta pesquisa, devem ser a base de qualquer planejamento em saúde, pois, por meio deles, as equipes podem acompanhar a evolução do tratamento e intervir em pontos estratégicos da cadeia de progressão da TB e HIV. Um registro completo e organizado possibilitaria a coordenação e gestão do cuidado e um trabalho mais integrado entre as especialidades que tratam o HIV e a TB o que, sem dúvida, instrumentalizaria mais as equipes nas tomadas de decisão, resultando em uma melhoria da qualidade dos serviços prestados, em menos iatrogenias, melhor prognóstico dos pacientes e melhora consequente da qualidade de vida.

Este trabalho tem como limitação a utilização de dados secundários manuscritos, como é o caso dos prontuários, utilizados pelos dois serviços que continham as informações dos pacientes. Assim, foram observadas lacunas no preenchimento de algumas informações e, ainda, prontuários faltantes, o que pode ter prejudicado a consistência interna do estudo.

\section{Conclusão}

O estudo contribuiu para o avanço do conhecimento no que tange à prevalência da ILTB em um serviço referência para PVHA, encontrando associação à infecção, ao sexo masculino, à condição prisional e ao número mais elevado de linfócitos T. Alguns casos progrediram para TB ativa, o que reforça a importância de um acompanhamento longitudinal e sistemático dos casos de ILTB, a fim de evitar tal desfecho; a adoção da terapia com isoniazida associada aos antirretrovirais, o mais precocemente possível, sustentada pela regularidade na ingestão desta medicação, traz perspectivas de qualidade de vida aos pacientes e maior taxa de sobrevida.

Sobre esses aspectos, observou-se que a terapia com isoniazida, embora recomendada, foi concluída em menos da metade dos pacientes, o que deve ser repensando pela organização dos serviços de saúde. Novas ferramentas são necessárias no sentido de motivar as PVHA a concluírem o seu tratamento, considerando-se que é uma população em alto risco de adoecimento e morte. Dentre os que tiveram o diagnóstico de TB ativa, mais da metade aderiu ao tratamento, o que é interessante, não obstante estes números estejam bem abaixo das perspectivas da OMS.

Os resultados evidenciados por este trabalho podem estar associados aos altos índices de mortalidade no cenário estudado e apontam para a relevância da priorização da busca de ILTB em PVHA, entendida como um desafio aos sistemas de saúde, que ainda não adotaram esta prática em seus programas. O estudo serve como uma referência sobre como é possível avaliar a progressão da condição de saúde de PVHA e elevar assim a qualidade dos serviços prestados a esta clientela, evidenciando alguns itens que no futuro podem vir a compor o elenco de indicadores obrigatórios, considerados para a avaliação dos programas de TB e de HIV/AIDS. Uma limitação é que muitos registros não foram preenchidos, o que denota o desafio dos profissionais em adotar esse recurso como objeto de avaliação e planejamento das ações em saúde. Este trabalho evidencia questões importantes em termos da organização dos serviços de saúde para a superação da problemática que é a mortalidade por AIDS e TB. 


\section{Colaboradores}

D. T. Santos participou da elaboração do projeto de pesquisa, coleta de dados, analises estatísticas, resultados, discussão e conclusão do artigo. M. C. Garcia participou da revisão de análise estatística bivariada e regressão múltipla. A. A. N. F. Costa participou da elaboração e revisão do projeto de pesquisa, elaboração do instrumento de coleta de dados e análises descritivas. F. M. Pieri participou da revisão textual, coleta de dados e redação final. D. A. P. Meier e S. P. R. Albanese participaram da revisão bibliográfica, coleta de dados, análises descritivas e revisão textual. R. A. Arcêncio contribuiu com a orientação e supervisão das análises estatísticas e revisão bibliográfica, revisão e correção da versão final do artigo. E. M. Dessunti contribuiu com a orientação e supervisão geral de todas as etapas do projeto de pesquisa e elaboração do artigo. Todos os autores aprovaram a versão final do artigo.

\section{Referências}

1. Rockwood N, Wilkinson RJ. Understanding and intervening in HIV-associated tuberculosis. Clin Med (Lond) 2015; 15 Suppl 6:s43-49.

2. Murray C, Ortblad K, Guinovart C, Lim SS, Wolock MT, Roberts DA, et al. Global, regional, and national incidence and mortality for HIV, tuberculosis, and malaria during 19902013: a systematic analysis for the Global Burden of Disease Study 2013. Lancet 2014; 384:1005-70.

3. World Health Organization. Global Tuberculosis Report 2015. http://www.who.int/tb/ publications/global_report/en/ (acessado em 01/Mar/2016).

4. Secretaria de Vigilância em Saúde, Ministério da Sáude. Boletim Epidemiológico 2014; 44(2):1-13.

5. Cailleaux-Cezar M. Diagnóstico e tratamento da tuberculose latente. Pulmão RJ 2012; 21:41-5.

6. Shayo GA, Moshiro C, Aboud S, Bakari M, Mugus FM. Acceptability and adherence to Isoniazid preventive therapy in HIV-infected patients clinically screened for latent tuberculosis in Dar es Salaam, Tanzania. BMC Infect Dis $2015 ; 15: 368-76$.

7. Akolo C, Adetifa I, Shepperd S, Volmink J. Treatment of latent tuberculosis infection in HIV-infected persons. Cochrane Database Syst Rev 2010; (1):CD000171.

8. World Health Organization. Guidelines for intensified tuberculosis case-finding and isoniazid preventive therapy for people living with HIV in resource constrained settings. Geneva: World Health Organization; 2011.

9. Pai M, Denkinger CM, Kik SV, Rangaka MX, Zwerling A, Oxlade O, et al. Gamma interferon release assays for detection of Mycobacterium tuberculosis infection. Clin Microbiol Rev 2014; 27:3-20.

10. Lester R, Hamilton R, Charalambous S, Dwadwa T, Chandler C, Churchyard GJ, et al. Barriers to implementation of isoniazid preventive therapy in HIV clinics: a qualitative study. AIDS 2010; 24 Suppl 5:S45-8. 
11. Aquino DS, Moura LCRV, Maruza M, Silva AP, Ximenes RAA, Lacerda HR, et al. Factors associated with treatment for latent tuberculosis in persons living with HIV/AIDS. Cad Saúde Pública 2015; 31:2505-13.

12. Meijerink H, Wisaksana R, Lestari M, Meilana I, Chaidir L, van der Ven AJ. Active and latent tuberculosis among HIV-positive injecting drug users in Indonesia. J Int AIDS Soc 2015; 18:19317.

13. Fekadu S, Teshome W, Alemu G. Prevalence and determinants of tuberculosis among HIV infected patients in south Ethiopia. J Infect Dev Ctries 2015; 9:898-904.

14. Ai JW, Ruan QL, Liu QH, Zhang WH. Updates on the risk factors for latent tuberculosis reactivation and their managements. Emerg Microbes Infect 2016; 5:e10.

15. Cohen MS, Chen YQ, McCauley M. Prevention of HIV-1 infection with early antiretroviral therapy. N Engl J Med 2011; 365:493-505.

16. Gordis L. Epidemiology. Philadelphia: Elsevier/Saunders; 2014.

17. Secretaria de Estado da Saúde do Paraná. Boletim Epidemiológico HIV/AIDS 2015; (2).

18. Secretaria de Vigilância em Saúde, Ministério da Saúde. Manual de recomendações para o controle da tuberculose no Brasil. Brasília: Ministério da Saúde; 2011.

19. Kussen GM, Dalla-Costa LM, Rossoni A, Raboni SM. Interferon-gamma release assay versus tuberculin skin test for latent tuberculosis infection among HIV patients in Brazil. Braz J Infect Dis 2016; 20:69-75.

20. Siqueira KZ, Mendonça SA, Penedo CC. Indicação da prova tuberculínica e infecção latente da tuberculose em HIV-positivos. Município de Blumenau, Estado de Santa Catarina, Brasil, 2004-2009. Epidemiol Serv Saúde 2012; 4:635-44.

21. Moura LCRV, Ximenes RAA, Lacerda HR, Miranda-Filho DB, Barbosa MT, Byington MR, et al. Predictive factors for repetition of the tuberculin test after a nonreactive test in patients with HIV/AIDS. Rev Panam Salud Pública 2012; 31:121-8
22. James PM, Ganaie FA, Kadahalli RL. The performance of quantiferon-TB gold in-tube (QFT-IT) test compared to tuberculin skin test (TST) in detecting latent tuberculosis infection (LTBI) in the presence of HIV coinfection in a high TB-burden area with BCG-vaccinated population. J Int Assoc Provid AIDS Care 2014; 13:47-55.

23. Parrella R, Esposito V, Onofrio M, Parrella G, Viglietti R, Sangiovanni V, et al. Interferon gamma release assays and tuberculin skin test performance in different settings of HIV immunodeficiency. In Vivo 2015; 29:137-40.

24. Departamento de Vigilância das Doenças Transmissíveis, Secretaria de Vigilância em Saúde, Ministério da Saúde. Panorama da tuberculose no Brasil: indicadores epidemiológicos e operacionais. Brasília: Ministério da Saúde; 2014.

25. Ayele HT, Mourik MSM, Debray TPA, Bonten MJM. Isoniazid prophylactic therapy for the prevention of tuberculosis in HIV infected adults: a systematic review and meta-analysis of randomized trials. PLoS One 2015; 10:e0142290.

26. Boon SD, Matteelli A, Ford N, Getahun H. Continuous isoniazid for the treatment of latent tuberculosis infection in people living with HIV: a systematic review and meta-analysis. AIDS 2016; 5:797-801.

27. Lee SH. Diagnosis and treatment of latent tuberculosis infection. Tuberc Respir Dis (Seoul) 2015; 78:56-63.

28. Rangaka MX, Cavalcante SC, Marais BJ, Thim S, Martinson NA, Swaminathan S, et al. Controlling the seedbeds of tuberculosis: diagnosis and treatment of tuberculosis infection. Lancet 2015; 386:2344-53.

29. Prevention Committee of the Japanese Society for Tuberculosis; Treatment Committee of the Japanese Society for Tuberculosis. Treatment guidelines for latent tuberculosis infection. Kekkaku 2014; 89:21-37. 
30. Centers for Disease Control and Prevention Revised surveillance case definition for HIV infection. MMWR Recomm Rep 2014; 63(RR03):1-10.

31. Chkhartishvili N, Kempker RR, Dvali N, Abashidze L, Sharavdze L, Gabunia P, et al. Poor agreement between interferon-gamma release assays and the tuberculin skin test among HIV-infected individuals in the country of Georgia. BMC Infect Dis 2013; 13:513.

32. Getahun H, Gunneberg C, Granich R, Nunn P. HIV infection-associated tuberculosis: the epidemiology and the response. Clin Infect Dis 2010; 50 Suppl 3:S201-7.

33. Eldred LJ, Churchyard G, Durovni B, Godfrey-Faussett P, Grant AD, Getahun H, et al. Isoniazid preventive therapy for HIV-infected people: evidence to support implementation. AIDS 2010; 24 Suppl 5:S1-3.

34. de Faria Gomes NM, da Mota Bastos MC, Marins RM, Barbosa AA, Soares ACP, de Oliveira Wilken de Abreu AM, et al. Differences between risk factors associated with tuberculosis treatment abandonment and mortality. Pulm Med 2015:546106.

35. Balasundaram A, Sarkar S, Hamide A, Lakshminarayanan S. Socioepidemiologic profile and treatment-seeking behavior of HIV/AIDS patients in a tertiary-care hospital in south India. J Health Popul Nutr 2014; 32:587-94.

36. Griffith DM. "I AM a Man": manhood, minority men's health and health equity. Ethn Dis 2015; 25:287-93.

37. Basu S, Stuckler D, McKee M. Addressing institutional amplifiers in the dynamics and control of tuberculosis epidemics. Am J Trop Med Hyg 2011; 84:30-7.

38. Al-Darraji HAA, Kamarulzaman A, Altice FL. Latent tuberculosis infection in a Malaysian prison: implications for a comprehensive integrated control program in prisons. BMC Public Health 2014; 10:14-21.
39. Marco A, Solé N, Orcau A, Escribano M, Del Baño L, Quintero S, et al. Prevalence of latent tuberculosis infection in inmates recently incarcerated in a men's prison in Barcelona. Int J Tuberc Lung Dis 2012; 1:60-4.

40. Ritter C, Elger BS. Prevalence of positive tuberculosis skin tests during 5 years of screening in a Swiss remand prison. Int J Tuberc Lung Dis 2012; 16:65-9.

41. Lemos AC, Matos ED, Bittencourt CN. Prevalence of active and latent TB among inmates in a prison hospital in Bahia, Brazil. J Bras Pneumol 2009; 35:63-8.

42. Carbone AS, Paião DS, Sgarbi RV, Lemos EF, Cazanti RF, Ota MM, et al. Active and latent tuberculosis in Brazilian correctional facilities: a cross-sectional study. BMC Infect Dis 2015; 22:15-24.

43. Nogueira PA, Abrahão RM, Galesi VM. Tuberculosis and latent tuberculosis in prison inmates. Rev Saúde Pública 2012; 46:119-27.

44. Encinales L, Zuñiga J, Yunis M, GranadosMontiel J, Granados J, Almeciga I, et al. Humoral immunity in tuberculin skin test anergy and its role in high-risk persons exposed to active tuberculosis. Mol Immunol 2010; 47:1066.

45. Günthard HF, Aberg JA, Eron JJ, Hoy JF, Telenti A, Benson CA. Antiretroviral treatment of adult HIV infection: 2014 recommendations of the International Antiviral Society-USA Panel. JAMA 2014; 312:410-25. 


\section{Abstract}

The study proposed to identify the prevalence of latent tuberculosis infection (LTBI) in persons living with HIV/AIDS (PLWHA), associated factors, and progression to active tuberculosis among the identified cases. This was an epidemiological and descriptive study. The study population consisted of PLWHA seen from 2003 and 2014 in a reference center for HIV/AIDS. Data were collected from patient files and the Brazilian Information System for Notifiable Diseases (SINAN). Bivariate statistical analysis used the chi-square test in which variables with $p<0.2$ were selected to enter the multiple regression model. Type I error was set at $5 \%(p<0.05)$ for all the tests. In the study, 690 cases were analyzed, and 66 (9.4\%) had a diagnosis of LTBI, with a prevalence of 7.5 cases per 100 patients. Of the 53 cases (80.3\%) of LTBI who were prescribed treatment with isoniazid, only 26 (39.4\%) concluded treatment, and $10(15.1 \%)$ dropped out. Male gender (adjusted $O R=1.8$; 95\% CI: 1.1-3.3), current incarceration (adjusted $O R=7.6$; 95\%CI: 2.35-24.9), and high lymphocyte count were associated with LTBI diagnosis (adjusted $O R=1.1$; 95\%CI: 1.1-1.2). Forty-seven (6.7\%) of LTBI cases progressed to active TB. Diagnosis and treatment of LTBI in PLWHA were not prioritized, which contributed to the development of active disease among cases. The study contributed to knowledge on LTBI in PLWHA, demonstrating crucial aspects in the management of PLWHA and the importance of detecting LTBI and early initiation of isoniazid, aimed at improved quality of life and prognosis for PLWHA.

Tuberculin Test; Latent Tuberculosis; Tuberculosis; Acquired Immunodeficiency Syndrome; HIV

\section{Resumen}

Se propone averiguar la prevalencia de infección latente por tuberculosis (ILTB) entre personas viviendo con VIH/SIDA (PVVS), sus factores asociados, $y$ si entre los casos identificados hubo progresión hacia la tuberculosis activa. Se trata de un estudio epidemiológico y descriptivo. La población estaba compuesta de PVVS, atendidas entre 2003 y 2014 en un centro de referencia para VIH/SIDA. Los datos fueron recogidos en base a historiales clínicos y fichas del Sistema Brasileiro de Información de Enfermedades de Notificación Obligatoria (SINAN). Se procedió a una estadística bivariada, con aplicación del test chi-cuadrado, donde las variables con valores de $p<0,2$ fueron seleccionadas para entrar en el modelo de regresión múltiple. Se fijó en todos los tests el error tipo I en un 5\% ( $p<$ 0,05). En el estudio, se analizaron 690 casos, donde 66 (un 9,4\%) presentaron el diagnóstico de ILTB, teniendo una prevalencia de 7,5 casos para cada 100 pacientes. De los $53(80,3 \%)$ casos de ILTB que tuvieron el tratamiento indicado con isoniazida, sólo $26(39,4 \%)$ lo concluyeron y $10(15,1 \%)$ lo abandonaron. Se observó que las variables sexo masculino (OR ajustado = 1,8; IC95\%: 1,1-3,3), situación en régimen de prisión (OR ajustado $=$ 7,6; IC95\%: 2,35-24,9) y un cómputo de linfocitos más altos son factores asociados al diagnóstico de ILTB (OR ajustado = 1, 1; IC95\%: 1,1-1,2). Se verificó que $47(6,7 \%)$ de los casos de ILTB progresaron hacia una tuberculosis activa. El diagnóstico y el tratamiento de ILTB en las PVVS no fueron priorizados, lo que contribuyó al desarrollo de la enfermedad activa entre los casos. El trabajo contribuyó al avance del conocimiento acerca de la ILTB entre PVVS, demostrando aspectos cruciales en lo que atañe al manejo de PVVS, e incluso la importancia de la detección de ILTB y la administración precoz de la isoniazida, con el fin de mejorar la calidad de vida y el pronóstico de las PVVS.

Prueba de Tuberculina; Tuberculosis Latente; Tuberculosis; Sindrome de Inmunodeficiencia Adquirida; VIH
Recebido em 28/Mar/2016

Versão final reapresentada em 14/Out/2016 Aprovado em 25/Out/2016 